\title{
ADENOCARCINOMA E TUMOR ESTROMAL (GIST) SINCRÔNICOS NO ESTÔMAGO: UMA RARA OCORRÊNCIA
}

\author{
SYNCHRONOUS ADENOCARCINOMA AND STROMAL TUMOR (GIST) IN THE \\ STOMACH: A RARE OCCURRENCE
}

\author{
Marcelo Amade Camargo ${ }^{1}$; Nelson Adami Andreollo, TCBC-SP ${ }^{2}$; Thais Cristina Hatsumura1; \\ Luiz Roberto Lopes, TCBC-SP ${ }^{3}$; Luciana Rodrigues Meirelles ${ }^{4}$
}

\section{INTRODUÇÃO}

A ocorrência de tumores primários múltiplos no estômago não é rara, a incidência varia entre 2 a $3 \%$, sendo a maioria dos casos composta por adenocarcinomas múltiplos ou associação entre adenocarcinoma e linfoma (MALT) ${ }^{1}$. Entretanto, adenocarcinoma e tumor mesenquimal (GIST) sincrônicos é muito raro, com apenas alguns relatos de casos na literatura ${ }^{2}$.

Este artigo descreve um caso raro de associação de adenocarcinoma gástrico e GIST, que apresentou recidiva tardia.

\section{RELATO DO CASO}

Homem, negro, 72 anos, portador de diabetes mellitus e hipertensão arterial sistêmica, referindo dor epigástrica e inapetência, além de emagrecimento de $14 \mathrm{~kg}$ em dois anos. Negava tabagismo e alcoolismo. Apresentava-se emagrecido e com mucosas descoradas. Não havia tumoração abdominal palpável.

Os exames laboratoriais demonstraram anemia $(\mathrm{Hb}=$ $6,0 \mathrm{~g} / \mathrm{dl}$ ). À endoscopia digestiva alta, visualizou-se lesão ulcerada de $5 \mathrm{~cm}$ de extensão, em incisura angularis, estendendo-se em pequena curvatura e parede posterior do corpo gástrico, compatível com neoplasia gástrica Borrmann III, cuja biópsia mostrou ser adenocarcinoma gástrico tubular moderadamente diferenciado, $H$. pylori negativo.

A tomografia computadorizada e a ultrassonografia abdominal foram utilizadas para o estadiamento e foram observados apenas dois nódulos próximos ao pâncreas e duodeno, sugestivos de linfonodomegalia.

Durante a operação, além da lesão descrita, foi palpada outra tumoração na grande curvatura, no corpo do estômago, de aproximadamente $3 \mathrm{~cm}$ de diâmetro. Foi também constatada linfonodomegalia infrapilórica. Realizada gastrectomia subtotal a $2 / 3$, ressecando-se as duas lesões, deixando margem de segurança de $5 \mathrm{~cm}$, além de linfadenectomia D2 e reconstrução em Y de Roux. A evolução pós-operatória foi satisfatória.

O exame anatomopatológico da peça cirúrgica demonstrou a presença de um adenocarcinoma tubular com áreas de padrão mucoso extracelular, moderadamente diferenciado, infiltrando a camada muscular própria (Figura 1) e um tumor estromal da parede gástrica, cujo perfil imunohistoquímico foi compatível com GIST (Figura 2), com alto índice mitótico (18 mitoses / 50 CGA) (Tabela 1). Havia metástase do adenocarcinoma para 1 de 28 linfonodos dissecados e as margens cirúrgicas estavam amplas e livres de neoplasia.

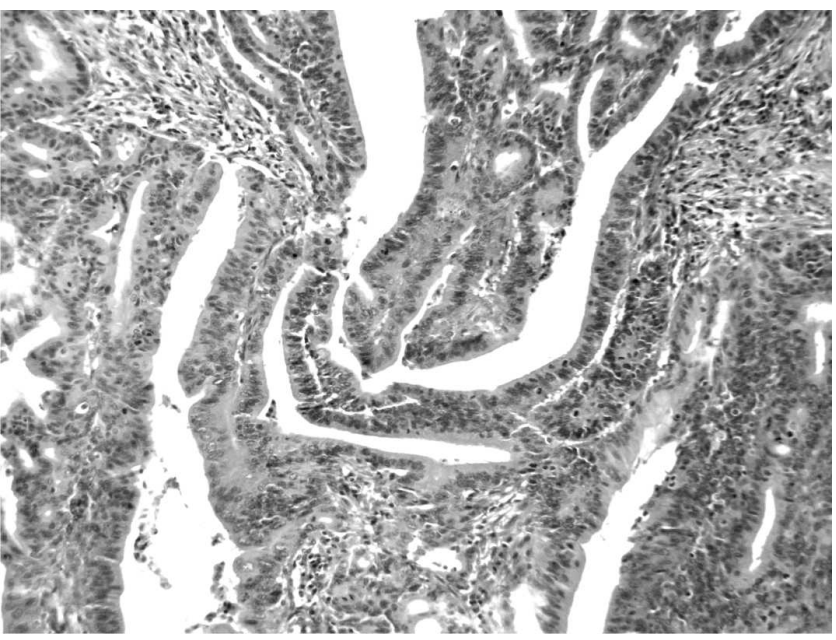

Figura 1 - Fotomicrografia de fragmento do adenocarcinoma tubular.

\footnotetext{
1. Residente de Cirurgia da Disciplina de Moléstias do Aparelho Digestivo do Departamento de Cirurgia - Departamento de Anatomia Patológica e Gastrocentro - Faculdade de Ciências Médicas da Universidade Estadual de Campinas (UNICAMP) - Campinas -SP

2. Professor Titular do Departamento de Cirurgia da Faculdade de Ciências Médicas da Universidade Estadual de Campinas (UNICAMP) - Campinas $-\mathrm{SP}$.

3. Professor Assistente; Doutor do Departamento de Cirurgia da Faculdade de Ciências Médicas da Universidade Estadual de Campinas (UNICAMP) - Campinas -SP.

4. Professora Assistente Doutora do Departamento de Anatomia Patológica Faculdade de Ciências Médicas da Universidade Estadual de Campinas (UNICAMP) - Campinas -SP.
}

Trabalho realizado na Disciplina de Moléstias do Aparelho Digestivo do Departamento de Cirurgia, Departamento de Anatomia Patológica e Gastrocentro - Faculdade de Ciências Médicas da Universidade Estadual de Campinas (UNICAMP) - Campinas -SP. 


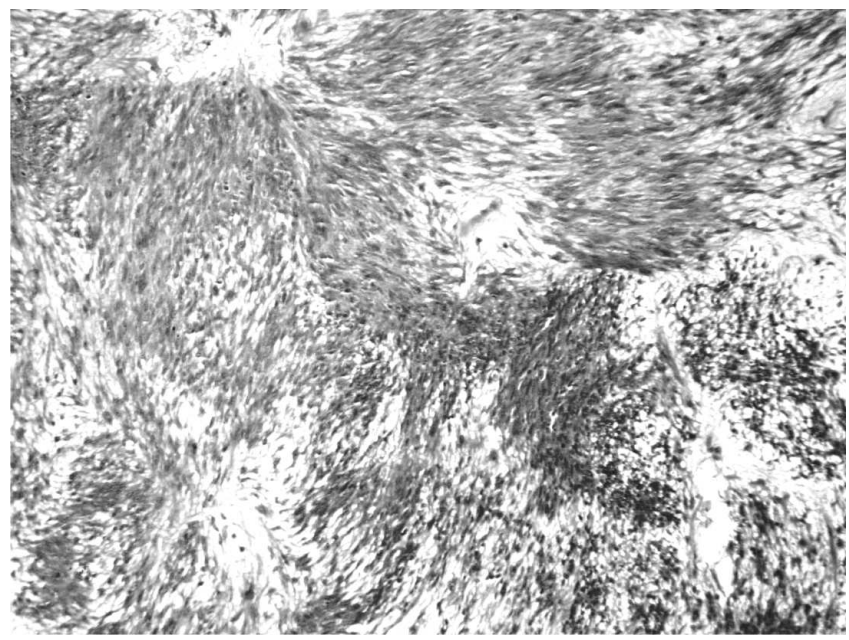

Figura 2 - Fotomicrografia de fragmento do GIST.

O acompanhamento pós-operatório foi feito com ultrassonografia, radiografias de tórax e dosagem de antígeno cárcinoembrionário (CEA) semestralmente. Dezessete meses após a operação, ocorreu elevação progressiva nos valores séricos de CEA. Nova tomografia computadorizada de abdome evidenciou uma tumoração medindo $7 \mathrm{~cm}$, adjacente à grande curvatura do estômago remanescente, próxima ao rim esquerdo.

$\mathrm{Na}$ laparotomia foi encontrada tumoração no espaço retro-gástrico, de aproximadamente $10 \mathrm{~cm}$ de diâmetro, pouco aderida ao mesocólon, que foi completamente removida. A análise histopatológica evidenciou um sarcoma fusocelular, cujo perfil imunohistoquímico corroborou o diagnóstico de GIST (Tabela 1).

O doente mantém-se em seguimento ambulatorial, assintomático e com exames normais 18 meses após a última cirurgia.

\section{DISCUSSÃO}

Tumores epiteliais e estromais concomitantes no estômago é evento raro. Maiorana et $a{ }^{2}{ }^{2}$ relatam que até 2000 haviam sido descritos cerca de 30 doentes com associação destes dois tumores e, narram a ocorrência de mais seis casos entre 2035 doentes operados com adenocarcinoma gástrico, sendo a freqüência da associação $0,29 \%$.

Liu et $a l .{ }^{3}$ relataram mais um caso, entretanto, referem tratar-se de tumor gástrico de "colisão", isto é, tumores justapostos sem tecido gástrico normal entre eles. Kaffles et $a l .{ }^{1}$ apresentam um doente em que foram diagnosticados, sincronicamente, adenocarcinoma, tumor estromal e linfoma.

A associação entre tumor gástrico epitelial e tumor estromal não tem etiopatogenia definida, apenas teorias baseadas em observações clínicas e modelos experimentais. Tada et al. ${ }^{4}$ observaram que o estômago que apresenta leiomiossarcoma tende a desenvolver lesões epiteliais malignas.

A hipótese mais aceita para justificar a ocorrência de tumores concomitantes é que um simples agente carcinogênico pode interagir em tecidos vizinhos, induzindo o desenvolvimento de tumores de tipos histopatológicos diferentes no mesmo órgão ${ }^{4}$.

No caso apresentado não foi proposta nenhum tipo de terapia adjuvante após a primeira cirurgia, por se tratar de doente idoso e a ressecção dos tumores considerada radical.

Atualmente a conduta para casos de recidiva do GIST é controversa. Acredita-se que, se existe condição para remoção cirúrgica do tumor recidivado, o mesmo deve ser realizado, pois o prognóstico do paciente, embora ainda tema de discussão, deverá ser melhor ${ }^{5}$.

Tabela 1 - Perfil Imunohistoquímico dos tumores estromais.

$1{ }^{a}$ Cirurgia CD 117: +/CD34:+/S100:-/1A4:+/AE1AE3:-/HHF35:-/Desmina:-/Actina:-

2a Cirurgia CD 117:+/ CD34:+/ S100:+/ 1A4:+

\begin{abstract}
A concomitant epithelial and stromal tumor in stomach is unusual in the literature. The purpose of this paper is to report the case and it's therapeutic management. A 72 year old black male patient, which upper digestive endoscopy showed a gastric neoplasm (Borrmann III) at incisura angularis and the biopsy revealed adenocarcinoma. A subtotal gastrectomy with D2 limphadenectomy and Roux-en-Y reconstruction was performed. The histopathology studies confirmed an adenocarcinoma and a gastric stromal tumor, whose immunohistochemical exam was compatible to GIST. Seventeen months after surgery, a computadorized tomography revealed a retrogastric tumor and laparotomy was indicated to remove the lesion (Rev. Col. Bras. Cir. 2008; 35(1): 061-063).
\end{abstract}

Key words: Adenocarcinoma; Adenosarcoma; Leiomyosarcoma; Neoplasms, multiple primary; Gastrointestinal Stromal Tumors; Gastrointestinal neoplasms; Stromal Cells. 


\section{REFERÊNCIAS}

1. Kaffes A, Hughes L, Hollinshead J, Katelaris P. Synchronous primary adenocarcinoma, mucosa-associated lymphoid tissue lymphoma and a stromal tumor in a Helicobacter pylori-infected stomach. J Gastroenterol Hepatol. 2002;17(9):1033-6.

2. MaioranaA, Fante R, Maria CesinaroA,Adriana Fano R. Synchronous occurrence of epithelial and stromal tumors in the stomach: a report of 6 cases. Arch Pathol Lab Med. 2000;124(5):682-6.

3. Liu SW, Chen GH, Hsieh PP. Collision tumor of the stomach: a case report of mixed Gastrointestinal stromal tumor and adenocarcinoma. J Clin Gastroenterol. 2002;35(4):332-4.

4. Tada 1, Zeze K, Nakashima K, Kuwahara A, Saitoh T, Kobayashi M. [Two cases of the independent coexistence of early cancer and leiomyosarcoma in the same stomach]. Gan No Rinsho. 1984;30:1812-18.
5. Camargo MA, Andreollo NA, Lopes LR, Coelho Neto JS, Stedile JA, Meirelles LR. Tumor estromal de estômago (GIST). ABCD Arq Bras Cir Dig. 2005;18(1):19-24.

Como citar este artigo:

Camargo MA, Andreollo NA, Hatsumura TC, Meirelles LR. Adenocarcinoma e tumor estromal (GIST) sincrônicos no estômago: uma rara ocorrência. Rev Col Bras Cir. [periódico na Internet] 2008; 35(1). Disponível em URL: http://www.scielo.br/rcbc

Endereço para correspondência:

Marcelo Amade Camargo

Rua Coronel Quirino, 1299 - apto 61.

Bairro Cambuí

13025-002 - Campinas - SP

E-mail: marcelocamargo@ sigmanet.com.br 